Check for updates

Cite this: RSC Adv., 2017, 7, 27900

\title{
Synthesis of $\alpha$-aminophosphonates using solvate ionic liquids $\uparrow$
}

\author{
Daniel J. Eyckens and Luke C. Henderson (iD)* \\ A range of $\alpha$-aminophosphonates were accessed in high yields and very rapidly, using solvate ionic liquids as \\ the reaction media. Reactions typically required less than 10 minutes to go to completion and precipitation \\ of these products into water excludes the use of traditional work up procedures, giving the products in very \\ high crude purity. Excellent functional group tolerance for both the aldehyde and amine reaction partners \\ was observed, and a range of bis-aminophosphonates derived from aromatic diamines were also accessed \\ in high yield and purity
}

Received 19th April 2017

Accepted 18th May 2017

DOI: $10.1039 / \mathrm{c} 7 \mathrm{ra0} 4407 \mathrm{k}$

rsc.li/rsc-advances

pose several problems: disposal of heavy metals and the reac-

\section{Introduction}

$\alpha$-Aminophosphonates are of great interest to organic chemists as they have vast applications in fields such as medicine, ${ }^{\mathbf{1 - 1 0}}$ agriculture, and in environmental decontamination. ${ }^{5,11,12}$ They are considered phosphorus analogues to $\alpha$-amino acids and the phosphorus' geometry has served as a useful tool in medicinal chemistry. ${ }^{13-16}$

The synthesis of these compounds via the Kabachnik-Fields or Pudovik reaction (Scheme 1) is relatively straight forward, using nucleophilic attack of di-alkyl or -aryl phosphite with an imine. ${ }^{12}$ The imine species can be generated in situ, as part of a three-component reaction, or preformed and added to the phosphite, both methodologies have been presented in the literature. ${ }^{12}$ Additionally, several reports employ excess of the amine or dialkyloxy-phosphite (in some cases both) which limits the broad appeal of this reaction.

A large amount of work has been focused on optimizing the synthesis of these molecules using a variety of Lewis acid catalysts, ${ }^{17,18}$ such as $\mathrm{CeCl}_{3}$-proline complex, ${ }^{19} \mathrm{GaI}_{3},{ }^{20} \mathrm{In}(\mathrm{OTf})_{3}$, $\mathrm{YbCl}_{3}, \mathrm{SmI}_{2}, \mathrm{MoO}_{2} \mathrm{Cl}_{2},{ }^{21} \mathrm{Sc}(\mathrm{OTf})_{3}$, and Dy(OTf) ${ }_{3}$ (ref. 12) among others. ${ }^{19,22}$ These efforts have given a variety of means to access these compounds, though the use of these Lewis acids always

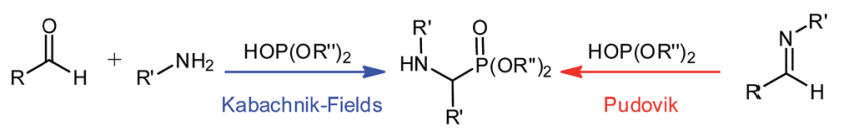

Scheme 1 Common approaches to the synthesis of $\alpha$ aminophosphonates.

Institute for Frontier Materials, Deakin University, 75 Pigdons Road, Geelong, Victoria, 3216, Australia. E-mail: luke.henderson@deakin.edu.au

$\dagger$ Electronic supplementary information (ESI) available. See DOI: $10.1039 / \mathrm{c} 7 \mathrm{ra} 04407 \mathrm{k}$ tion solvent, the high toxicity of these materials, their sensitivity to atmospheric conditions, and their expensive nature.

Ionic liquids (ILs), in their many forms, have been used in many areas of chemistry from materials science through to solvents to facilitate organic transformations. ${ }^{23-29}$ The use of ILs as solvents for this reaction have been reported with some excellent results, ${ }^{30-34}$ particularly for the pseudo-ionic liquid 5.0 M lithium perchlorate in dithethyl ether (5 M LPDE), or the use of $\mathrm{LiClO}_{4}$ as an additive to the reaction mixture. ${ }^{18,35-40}$ These reactions were able to isolate $\alpha$-aminophosphonates in very high yield using reaction times under 1 hour.

Despite these benefits, the use of 5.0 M LPDE as the reaction media experiences several limitations, which include: enhanced sensitivity to atmospheric conditions, problematic solvent preparation, inability to be heated (due to potential explosive nature of the $\mathrm{ClO}_{4}{ }^{-}$anion), and reagent restriction as not all reagents will be soluble in the pseudo-ionic liquid. Also, while the use of ionic liquids is an improvement over the use of metalbased catalysts, these procedures still use large amounts of organic solvent in the recovery and purification of the synthesised phosphonate. Accessing these compounds in high yield, short reaction time, and with minimal purification remains a challenge in organic synthesis.

We recently reported the use of solvate ionic liquids (SILs), being equimolar mixtures of LiTFSI in tri- or tetra-glyme (referred to as $\left[\mathbf{G}_{3}(\mathbf{L i})\right] \mathbf{T F S I}$ or $\left[\mathbf{G}_{\mathbf{4}}(\mathbf{L i})\right] \mathbf{T F S I}$, respectively), as suitable reaction media for electrocyclisation reactions and characterised them using Kamlet-Taft parameters (Fig. 1). ${ }^{\mathbf{4 1 4 2}}$

Indeed these solvate ionic liquids serve as a stable and easilyhandled surrogate to 5.0 M LPDE, while often giving superior reaction outcomes. They are able to be heated without concern, present minimal toxicity, ${ }^{43}$ are simple and cheap to produce, and can be stored over molecular sieves to maintain their anhydrous nature, if required. This work sought to determine if the synthesis of $\alpha$-aminophosphonates could be successfully 
carried out in solvate ionic liquids and if so, how does this compare to other ionic liquid and molecular solvents.

Herein we report the application of solvate ionic liquids as a solvent for the rapid and high yielding synthesis of $\alpha$-aminophosphonates in a three-component reaction. This reaction proceeds at room temperature in 5 minutes, with the majority of crude products being obtained by precipitation and are analytically pure, with minimal workup.

This work began by examining the Kabachnik-Fields reaction (i.e. three-component reaction) to minimize reagent handling and as it represents the more efficient reaction pathway compared to pre-synthesising the imine. As a model system for optimisation, benzaldehyde 1, aniline 2, and diphenyl phosphite were chosen (Table 1).

Initially, the three-component reaction was stirred at room temperature for 30 minutes in $\left[\mathbf{G}_{3}(\mathbf{L i})\right] \mathbf{T F S I}$, accessing pure $\mathbf{3}$ in a very promising yield of $82 \%$ (Table 1 , entry 1 ). In an effort to reduce reaction time, without sacrificing the yield, the reaction time was reduced to 5 minutes (Table 1, entry 2), giving a excellent yield of $87 \%$. Further reduction to 1 minute (Table 1 , entry 3 ) gave the desired product in a slightly depressed yield of $78 \%$. Using this, a 5 minute reaction was considered the optimal due to excellent yield and the reaction occurring on a very practical timescale. Repeating this reaction in $\left[\mathbf{G}_{\mathbf{4}}(\mathbf{L} \mathbf{i})\right]$ TFSI, gave the desired product in an excellent yield of $91 \%$ (Table 1, entry 4). Note that the isolation of these compounds was achieved via precipitation of the crude products into water, and not via chromatographic methods.

Nevertheless, with these conditions in hand, our attention turned to assessing the functional group tolerance of this reaction in SILs (Table 2). During the reaction scoping process, many aniline derivatives were observed to react extremely quickly, with some forming the solid product almost instantly, preventing further stirring.

The first reaction using these conditions and 4-nitroaniline proceeded in good yield for [ $\left.\mathbf{G}_{3}(\mathbf{L i})\right] \mathbf{T F S I}(64 \%)$ and poor yield in [G $\left.\mathbf{G}_{\mathbf{4}}(\mathbf{L i})\right]$ TFSI $(25 \%)$ (Table 2, entries 1 \& 2, respectively). The poor yield for the latter SIL was attributed to both the poor nucleophilicity of the aniline and the problematic isolation of the product, due to repeated recrystalisation. Interestingly, the introduction of a halogen-substitution on the aniline gave excellent yields, with both $\left[\mathbf{G}_{3}(\mathbf{L i})\right] \mathbf{T F S I}$ and $\left[\mathbf{G}_{4}(\mathbf{L i})\right] \mathbf{T F S I}$ fetching an exemplary yields of $96 \%$ each (Table 2 , entries $3 \& 4$, respectively). A similar yield was obtained from the electrondonating 4-aminophenol in $\left[\mathbf{G}_{3}(\mathbf{L i})\right] \mathbf{T F S I}(82 \%)$ and $\left[\mathbf{G}_{4}(\mathbf{L i})\right]$ TFSI (92\%) (Table 2, entries $7 \& 8$, respectively). Incorporating 4fluoroaniline into the given reaction conditions gave an excellent yield in $\left[\mathbf{G}_{3}(\mathbf{L i})\right] \mathbf{T F S I}(84 \%$, Table 2 , entry 7$)$. Though when
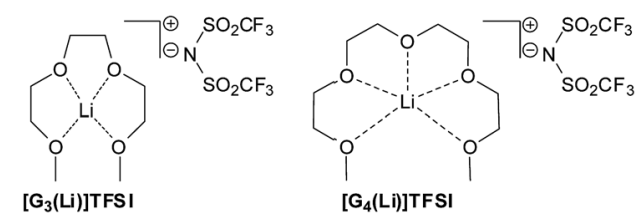

Fig. 1 Simplified structures of solvate ionic liquids used in this work.
Table 1 Optimisation of $\alpha$-aminophosphonate synthesis

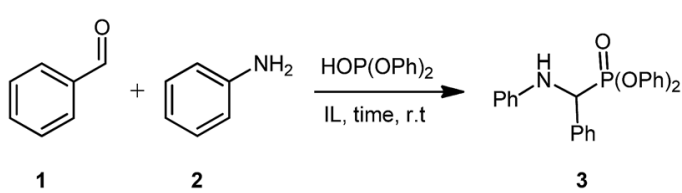

\begin{tabular}{llll}
\hline Entry & Solvent & Time $(\mathrm{min})$ & Yield $^{a}(\%)$ \\
\hline 1 & {$\left[\mathbf{G}_{3}(\mathbf{L i})\right] \mathbf{T F S I}$} & 30 & 82 \\
2 & {$\left[\mathbf{G}_{3}(\mathbf{L i})\right] \mathbf{T F S I}$} & 5 & 87 \\
3 & {$\left[\mathbf{G}_{3}(\mathbf{L i})\right]$ TFSI } & 1 & 78 \\
4 & {$\left[\mathbf{G}_{4}(\mathbf{L i})\right]$ TFSI } & 5 & 91 \\
${ }^{a}$ & &
\end{tabular}

[ $\left.\mathbf{G}_{4}(\mathbf{L i})\right]$ TFSI was used, a slightly diminished yield of $68 \%$ (Table 2, entry 8) was obtained. Similarly, 3-chloroaniline proceeded well in $\left[\mathbf{G}_{\mathbf{3}}(\mathbf{L i})\right] \mathbf{T F S I}(83 \%$, Table 2 , entry 9), a result not consistent in $\left[\mathbf{G}_{\mathbf{4}}(\mathbf{L i})\right] \mathbf{T F S I}(59 \%$, Table 2, entry 10). Exploring the effect of 3 -(trifluoromethyl)aniline in this reaction revealed a excellent yields of $86 \%$ and $81 \%$ in $\left[\mathbf{G}_{3}(\mathbf{L i})\right]$ TFSI and $\left[\mathbf{G}_{4}(\mathbf{L i})\right]$ TFSI, respectively (Table 2, entries $11 \& 12$, respectively). Introducing a bis-trifluoromethylaniline derivative demonstrated the robust nature of the reaction conditions, with both $\left[\mathbf{G}_{3}(\mathbf{L i})\right] \mathbf{T F S I}$ and $\left[\mathbf{G}_{4}(\mathbf{L i})\right] \mathbf{T F S I}$ proceeding in good yields of $54 \%$ and $60 \%$, respectively (Table 2, entries $13 \& 14$, respectively). It is worth noting as well that the isolation of some products from $\left[\mathbf{G}_{4}(\mathbf{L i})\right]$ TFSI was slightly more difficult than the $\left[\mathbf{G}_{3}(\mathbf{L i})\right] \mathbf{T F S I}$, whereby the isolated material contained traces of the glyme from the IL. The repeated precipitation to remove these traces of glyme may be responsible for the slightly reduced yield in certain

Table 2 Reaction scoping using substituted anilinic amines

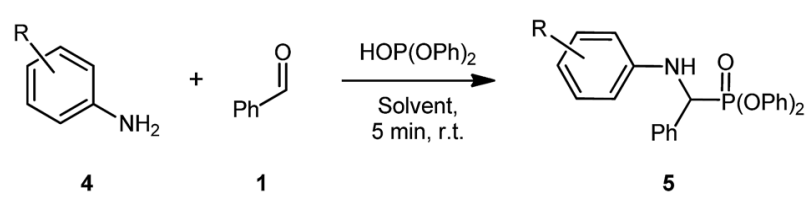

\begin{tabular}{|c|c|c|c|c|}
\hline Entry & Compound & $\mathrm{R}$ & Solvent & Yield $^{a}(\%)$ \\
\hline 1 & \multirow[t]{2}{*}{$5 a$} & \multirow[t]{2}{*}{$4-\mathrm{NO}_{2}$} & {$\left[\mathrm{G}_{\mathbf{3}}(\mathrm{Li})\right] \mathrm{TFSI}$} & 64 \\
\hline 2 & & & {$\left[\mathrm{G}_{4}(\mathrm{Li})\right] \mathrm{TFSI}$} & 25 \\
\hline 3 & \multirow[t]{2}{*}{$5 \mathbf{b}$} & \multirow[t]{2}{*}{$4-\mathrm{Cl}$} & {$\left[\mathrm{G}_{3}(\mathbf{L i})\right] \mathrm{TFSI}$} & 96 \\
\hline 4 & & & {$\left[\mathrm{G}_{4}(\mathrm{Li})\right] \mathrm{TFSI}$} & 96 \\
\hline 5 & \multirow[t]{2}{*}{$5 c$} & \multirow[t]{2}{*}{$4-\mathrm{OH}$} & {$\left[G_{3}(\mathbf{L i})\right]$ TFSI } & 82 \\
\hline 6 & & & {$\left[\mathrm{G}_{4}(\mathrm{Li})\right] \mathrm{TFSI}$} & 92 \\
\hline 7 & \multirow[t]{2}{*}{$5 d$} & \multirow[t]{2}{*}{$4-\mathrm{F}$} & {$\left[\mathrm{G}_{3}(\mathrm{Li})\right] \mathrm{TFSI}$} & 84 \\
\hline 8 & & & {$\left[\mathrm{G}_{4}(\mathrm{Li})\right] \mathrm{TFSI}$} & 68 \\
\hline 9 & \multirow[t]{2}{*}{$5 e$} & \multirow[t]{2}{*}{$3-\mathrm{Cl}$} & {$\left[\mathrm{G}_{3}(\mathrm{Li})\right] \mathrm{TFSI}$} & 83 \\
\hline 10 & & & {$\left[\mathrm{G}_{4}(\mathrm{Li})\right] \mathrm{TFSI}$} & 59 \\
\hline 11 & \multirow[t]{2}{*}{$5 f$} & \multirow[t]{2}{*}{$3-\mathrm{CF}_{3}$} & {$\left[\mathrm{G}_{3}(\mathrm{Li})\right] \mathrm{TFSI}$} & 86 \\
\hline 12 & & & {$\left[\mathrm{G}_{4}(\mathrm{Li})\right] \mathrm{TFSI}$} & 81 \\
\hline 13 & \multirow[t]{2}{*}{$5 g$} & \multirow[t]{2}{*}{$3,5-\mathrm{CF}_{3}$} & {$\left[\mathrm{G}_{3}(\mathrm{Li})\right]$ TFSI } & 54 \\
\hline 14 & & & {$\left[G_{4}(\mathrm{Li})\right] \mathrm{TFSI}$} & 60 \\
\hline
\end{tabular}

${ }^{a}$ Isolated yield. 
instances. With this final example of the reaction's high functional group tolerance, attention was then turned to the effect of aldehyde substitution (Table 3).

Varying the aldehyde component of the reaction, and maintaining the aniline, scoping began with the use of 4-bromobenzaldehyde, which proceeded in excellent yield of $90 \%$ and $91 \%$ in both $\left[\mathbf{G}_{3}(\mathbf{L i})\right] \mathbf{T F S I}$ and $\left[\mathbf{G}_{\mathbf{4}}(\mathbf{L i})\right] \mathbf{T F S I}$ (Table 3, entries 1 $\& 2$, respectively). Utilizing $p$-tolualdehyde also gave encouraging yields of $90 \%$ in $\left[\mathbf{G}_{3}(\mathbf{L i})\right] \mathbf{T F S I}$ and $86 \%$ in [G4(Li)]TFSI (Table 3, entries $3 \& 4$, respectively). Good yields of $69 \%$ and $59 \%$ were observed in $\left[\mathbf{G}_{3}(\mathbf{L i})\right] \mathbf{T F S I}$ and $\left[\mathbf{G}_{4}(\mathbf{L i})\right] \mathbf{T F S I}$, respectively, when using 4-nitrobenzaldehyde as the aldehyde source (Table 3, entries $5 \& 6$, respectively). The reaction also proceed well with the application of 4-fluorobenzaldehyde, obtaining the final product in good yields of $77 \%$ and $78 \%$ in $\left[\mathbf{G}_{3}(\mathbf{L i})\right] \mathbf{T F S I}$ and [G4(Li)]TFSI (Table 3, entries $7 \& 8$, respectively). The use of salicylaldehyde also showed good promise, giving pure product in $84 \%$ and $76 \%$ in $\left[\mathbf{G}_{3}(\mathbf{L i})\right] \mathbf{T F S I}$ and $\left[\mathbf{G}_{\mathbf{4}}(\mathbf{L i})\right] \mathbf{T F S I}$, respectively (Table 3, entries $9 \& 10$, respectively) and inclusion of a bissubstituted aldehyde (3,4-dichlorobenzaldehyde) further exemplified the ability of the SILs to assist this reaction, giving the product $\mathbf{7 f}$ in good yields of $74 \%$ in $\left[\mathbf{G}_{3}(\mathbf{L i})\right] \mathbf{T F S I}$ and $79 \%$ in $\left[\mathbf{G}_{4}(\mathbf{L i})\right]$ TFSI (Table 3 , entries $11 \& 12$, respectively). With this data in hand our attention turned to the examination of carrying out multiple reactions on bis-amines.

The reactions proceeded very smoothly within 5 minutes, giving the desired product for a range of functional groups. The yields were variable depending on the SIL and the electronics of the aldehyde. No discernable trend or difference was noted between either the $\left[\mathbf{G}_{3}(\mathbf{L i})\right] \mathbf{T F S I}$ or $\left[\mathbf{G}_{4}(\mathbf{L i})\right] \mathbf{T F S I}$, with similar yields given for each example presented (Table 4 ).

Table 3 Reaction scoping using substituted benzaldehydes

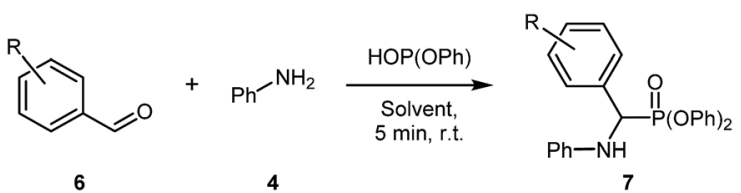

\begin{tabular}{|c|c|c|c|c|}
\hline Entry & Compound & $\mathrm{R}$ & Solvent & Yield $^{a}(\%)$ \\
\hline 1 & \multirow[t]{2}{*}{$7 a$} & \multirow[t]{2}{*}{$4-\mathrm{Br}$} & {$\left[\mathrm{G}_{3}(\mathrm{Li})\right] \mathrm{TFSI}$} & 90 \\
\hline 2 & & & {$\left[\mathrm{G}_{4}(\mathrm{Li})\right] \mathrm{TFSI}$} & 91 \\
\hline 3 & \multirow[t]{2}{*}{$7 \mathbf{b}$} & \multirow[t]{2}{*}{ 4-Me } & {$\left[\mathrm{G}_{3}(\mathrm{Li})\right] \mathrm{TFSI}$} & 90 \\
\hline 4 & & & {$\left[G_{4}(L i)\right]$ TFSI } & 86 \\
\hline 5 & \multirow[t]{2}{*}{$7 c$} & \multirow[t]{2}{*}{$4-\mathrm{NO}_{2}$} & {$\left[\mathrm{G}_{3}(\mathrm{Li})\right] \mathrm{TFSI}$} & 69 \\
\hline 6 & & & {$\left[\mathrm{G}_{4}(\mathrm{Li})\right] \mathrm{TFSI}$} & $59^{b}$ \\
\hline 7 & \multirow[t]{2}{*}{$7 d$} & \multirow[t]{2}{*}{$4-\mathrm{F}$} & {$\left[\mathrm{G}_{3}(\mathrm{Li})\right] \mathrm{TFSI}$} & 77 \\
\hline 8 & & & {$\left[G_{4}(\mathbf{L i})\right]$ TFSI } & 78 \\
\hline 9 & \multirow[t]{2}{*}{$7 e$} & \multirow[t]{2}{*}{$2-\mathrm{OH}$} & {$\left[G_{3}(L i)\right]$ TFSI } & 84 \\
\hline 10 & & & {$\left[G_{4}(L i)\right]$ TFSI } & 76 \\
\hline 11 & \multirow[t]{2}{*}{$7 f$} & \multirow[t]{2}{*}{$3,4-\mathrm{Cl}$} & {$\left[\mathrm{G}_{3}(\mathrm{Li})\right]$ TFSI } & 74 \\
\hline 12 & & & {$\left[\mathbf{G}_{\mathbf{4}}(\mathbf{L i})\right] \mathrm{TFSI}$} & 79 \\
\hline
\end{tabular}

${ }^{a}$ Isolated yield. ${ }^{b}$ This material possessed some of the $\alpha$ hydroxyphosphonate ( $17 \%$ by ${ }^{1} \mathrm{H}$ NMR) resulting from direct attack of the phosphite on 4-nitrobenzaldehyde.
Table 4 Synthesis of bis- $\alpha$-aminophosphonates

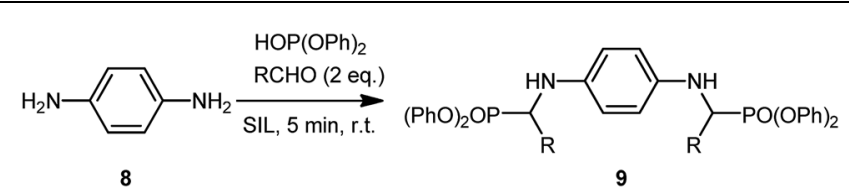

\begin{tabular}{lllll}
\hline Entry & Compound & $\mathrm{R}$ & Solvent & Yield $^{a}(\%)$ \\
\hline 1 & $9 \mathbf{a}$ & $\mathrm{Ph}$ & {$\left[\mathbf{G}_{3}(\mathbf{L i})\right]$ TFSI } & 64 \\
2 & & & {$\left[\mathbf{G}_{\mathbf{4}}(\mathbf{L i})\right]$ TFSI } & 52 \\
3 & $\mathbf{4 b}$ & $4-\mathrm{BrPh}$ & {$\left[\mathbf{G}_{3}(\mathbf{L i})\right]$ TFSI } & 36 \\
4 & & & {$\left[\mathbf{G}_{\mathbf{4}}(\mathbf{L i})\right]$ TFSI } & 65 \\
5 & $\mathbf{4}$ & $4-\mathrm{NO}_{2} \mathrm{Ph}$ & {$\left[\mathbf{G}_{3}(\mathbf{L i})\right]$ TFSI } & 18 \\
6 & & & {$\left[\mathbf{G}_{4}(\mathbf{L i})\right]$ TFSI } & 33 \\
${ }^{a}$ Isolated yield. & & &
\end{tabular}

Repeating this process but using the ortho- and metasubstituted diamino anilines (Scheme 2), gave varied results. As may be expected, the formation of $\mathbf{1 1}$ proceeded in a good but reduced yield in both $\left[\mathbf{G}_{3}(\mathbf{L i})\right] \mathbf{T F S I}$ or $\left[\mathbf{G}_{4}(\mathbf{L i})\right] \mathbf{T F S I}$ of $25 \%$ and $34 \%$, respectively. This was attributed to the increased steric hinderance imposed by the meta-substitution of the parent diamine 10. This was consistent with our observations for the formation of 13, possessing ortho-substitution where only $9 \%$ was isolated for $\left[\mathbf{G}_{3}(\mathbf{L i})\right] \mathbf{T F S I}$ and no product was able to be isolated when using $\left[\mathbf{G}_{\mathbf{4}}(\mathbf{L i})\right] \mathbf{T F S I}$.

A final aspect of this work which was considered challenging and had not been investigated previously is the synthesis of non-symmetric bis- $\alpha$-aminophosphonates, using two different aldehydes (Scheme 3). There is a considerable challenge in this procedure considering the speed at which the reaction takes place, thus controlling the regiochemistry will be difficult. Experimentally, the use of two aldehydes of similar electronic nature were chosen, reduce any bias in the reacting system, e.g. the use of a reactive aldehyde followed by an unreactive aldehyde would favour the formation of the non-symmetric product.

Therefore, in this case the first aldehyde was added to the solution of phosphite and amine in $\left[\mathbf{G}_{3}(\mathbf{L i})\right] \mathbf{T F S I}$, then after 5

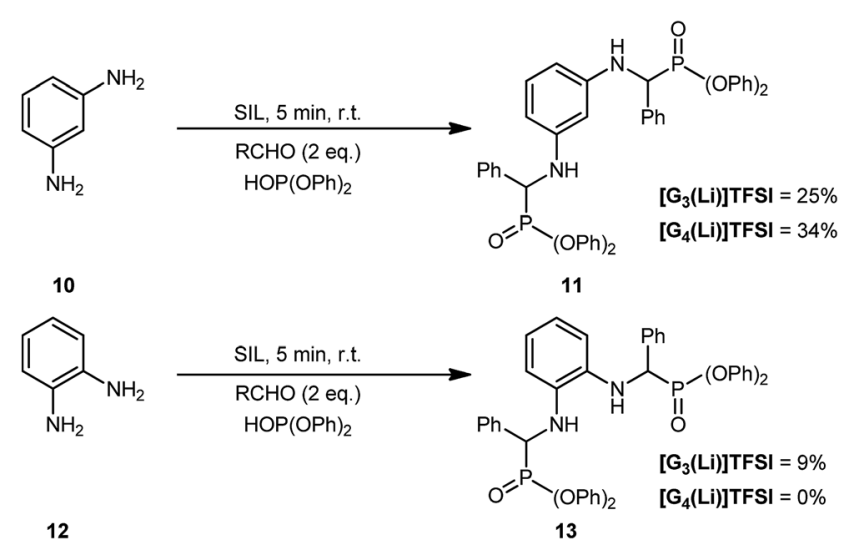

Scheme 2 Common approaches to the synthesis of $\alpha$ aminophosphonates. 


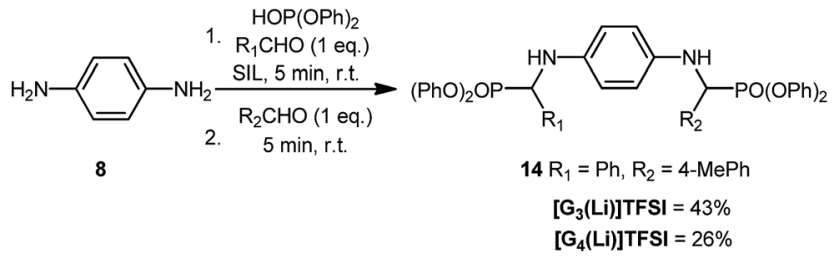

Scheme 3 Non-symmetric bis- $\alpha$-aminophosphonate synthesis.

minutes the second aldehyde was added. Using $p$-tolualdehyde and benzaldehyde, the target non-symmetric- $\alpha$-aminophosphonate 14 was successfully synthesised in a moderate yield of $43 \%$ in $\left[\mathbf{G}_{3}(\mathbf{L i})\right] \mathbf{T F S I}, 26 \%$ in [ $\left.\mathbf{G}_{4}(\mathbf{L i})\right] \mathbf{T F S I}$, and in only 10 minutes (Scheme 3). In each case the standard precipitation described was also successful, giving $\mathbf{1 4}$ in analytical purity.

\section{Representative experimental procedure}

A round bottom flask was charged with aldehyde $(1.00 \mathrm{mmol})$, which was dissolved in either $\left[\mathbf{G}_{3}(\mathbf{L i})\right] \mathbf{T F S I}$ or $\left[\mathbf{G}_{\mathbf{4}}(\mathbf{L i})\right] \mathbf{T F S I}(0.5$ $\mathrm{mL})$. Aniline $(1.00 \mathrm{mmol})$ was then added, before the addition of diphenyl phosphite $(0.230 \mathrm{~mL}, 1.20 \mathrm{mmol})$ and stirred at room temperature for the given time period. Diethyl ether $(10 \mathrm{~mL})$ was added at the conclusion of the reaction, before the addition of deionised water $(10 \mathrm{~mL})$ causing a fine precipitate to form. The removal of diethyl ether under reduced pressure afforded a suspension of precipitate in the aqueous phase, which was then filtered washing with excess water and petroleum spirits $\left(40-60{ }^{\circ} \mathrm{C}\right)$.

\section{Conclusions}

In conclusion, the use of solvate ionic liquids as excellent reaction media for the Kabachnik-Fields reaction has been shown. A wide range of $\alpha$-aminophosphonates were able to be synthesised in 5 minutes with simple precipitation giving the desired compound in $>95 \%$ purity. Extension of this methodology to bis- $\alpha$-aminophosphonates, using para-diaminobenzene was also successful, in the same 5 minute reaction duration and in high yield. Using ortho- and metadiaminobenzene gave the desired products but in lower yield (34\% and 9\%, respectively) presumably due to steric influences. Finally, synthesis of a non-symmetric bis- $\alpha$-aminophosphonate was achieved, using sequential addition of the aldehydes in excellent yield of $43 \%$. No discernible trend with respect to which SIL was optimal for a given reaction, though it was noted that removal of $\mathbf{G}_{\mathbf{4}}$ was more challenging compared to the shorter $\mathbf{G}_{3}$ analogue, this is presumably due to its slightly more 'organic' nature.

\section{Acknowledgements}

The authors would like to thank the Institute of Frontier Materials (IFM) for a postgraduate scholarship to DE.

\section{Notes and references}

1 N. Ali, S. Zakir, M. Patel and M. Farooqui, Eur. J. Med. Chem., 2012, 50, 39-43.

2 R. Damiche and S. Chafaa, J. Mol. Struct., 2017, 1130, 10091017.

3 L. Jin, B. Song, G. Zhang, R. Xu, S. Zhang, X. Gao, D. Hu and S. Yang, Bioorg. Med. Chem. Lett., 2006, 16, 1537-1543.

4 I. H. Kim, Y. K. Park, H. Nishiwaki, B. D. Hammock and K. Nishi, Bioorg. Med. Chem., 2015, 23, 7199-7210.

5 A. Mucha, P. Kafarski and L. Berlicki, J. Med. Chem., 2011, 54, 5955-5980.

6 Q. Wang, M. Zhu, R. Zhu, L. Lu, C. Yuan, S. Xing, X. Fu, Y. Mei and Q. Hang, Eur. J. Med. Chem., 2012, 49, 354-364.

7 K. P. Boroujeni, E. R. Shirazi and M. M. Doroodmand, Phosphorus, Sulfur Silicon Relat. Elem., 2015, 191, 683-688.

8 L. Maier, Phosphorus, Sulfur Silicon Relat. Elem., 1990, 53, 4367.

9 C. Sampath, P. Harika and N. Revaprasadu, Phosphorus, Sulfur Silicon Relat. Elem., 2015, 191, 1081-1085.

10 C. Subramanyam, S. Thaslim Basha, G. Madhava, S. Nayab Rasool, S. Adam, S. Durga Srinivasa Murthy and C. Naga Raju, Phosphorus, Sulfur Silicon Relat. Elem., 2017, 192, 267-270.

11 E. D. Naydenova, P. T. Todorov and K. D. Troev, Amino Acids, 2010, 38, 23-30.

12 G. Keglevich and E. Balint, Molecules, 2012, 17, 12821-12835.

13 J. Joossens, P. Van der Veken, G. Surpateanu, A.-M. Lambeir, I. El-Sayed, O. M. Ali, K. Augustyns and A. Haemers, J. Med. Chem., 2006, 49, 5785-5793.

14 D. Hocková, D. T. Keough, Z. Janeba, T.-H. Wang, J. de Jersey and L. W. Guddat, J. Med. Chem., 2012, 55, 6209-6223.

15 J. W. De Schutter, J. Park, C. Y. Leung, P. Gormley, Y.-S. Lin, Z. Hu, A. M. Berghuis, J. Poirier and Y. S. Tsantrizos, J. Med. Chem., 2014, 57, 5764-5776.

16 Y. Wu, C. J. Aquino, D. J. Cowan, D. L. Anderson, J. L. Ambroso, M. J. Bishop, E. E. Boros, L. Chen, A. Cunningham, R. L. Dobbins, P. L. Feldman, L. T. Harston, I. W. Kaldor, R. Klein, X. Liang, M. S. McIntyre, C. L. Merrill, K. M. Patterson, J. S. Prescott, J. S. Ray, S. G. Roller, X. Yao, A. Young, J. Yuen and J. L. Collins, J. Med. Chem., 2013, 56, 5094-5114.

17 Y. Cai, Y. Li, M. Zhang, J. Fu and Z. Miao, $R S C A d v .$, 2016, 6, 69352-69356.

18 N. Azizi and M. R. Saidi, Eur. J. Org. Chem., 2003, 2003, 46304633.

19 C. D. G. da Silva, A. R. Oliveira, M. P. D. Rocha, R. Katla, E. R. Botero, É. C. da Silva and N. L. C. Domingues, $R S C$ $A d v .$, 2016, 6, 27213-27219.

20 P. Sun, Z. Hu and Z. Huang, Synth. Commun., 2004, 34, 42934299.

21 R. G. de Noronha, C. C. Romão and A. C. Fernandes, Catal. Commun., 2011, 12, 337-340.

22 S. Bhagat and A. K. Chakraborti, J. Org. Chem., 2007, 72, 1263-1270. 
23 K. M. Beggs, M. D. Perus, L. Servinis, L. A. O'Dell, B. L. Fox, T. R. Gengenbach and L. C. Henderson, RSC Adv., 2016, 6, 32480-32483.

24 L. C. Henderson and N. Byrne, Green Chem., 2011, 13, 813816.

25 L. C. Henderson, M. T. Thornton, N. Byrne, B. L. Fox, K. D. Waugh, J. S. Squire, L. Servinis, J. P. Delaney, H. L. Brozinski, L. M. Andrighetto and J. M. Altimari, C. $R$. Chim., 2013, 16, 634-639.

26 T. T. Megan, C. H. Luke, B. Nolene and M. P. Frederick, Curr. Org. Chem., 2012, 16, 121-126.

27 N. Debeljuh, C. J. Barrow, L. Henderson and N. Byrne, Chem. Commun., 2011, 47, 6371-6373.

28 M. Maghe, C. Creighton, L. C. Henderson, M. G. Huson, S. Nunna, S. Atkiss, N. Byrne and B. L. Fox, J. Mater. Chem. A, 2016, 4, 16619-16626.

29 T. Welton, Chem. Rev., 1999, 99, 2071-2083.

30 T. Olszewski, Synthesis, 2014, 46, 403-429.

31 J. S. Yadav, B. V. S. Reddy and P. Sreedhar, Green Chem., 2002, 4, 436-438.

32 K. P. Boroujeni, E. R. Shirazi and M. M. Doroodmand, Phosphorus, Sulfur Silicon Relat. Elem., 2016, 191, 683-688.

33 D. Fang, J. Yang and C. Ni, Heteroat. Chem., 2011, 22, 5-10.
34 R. Hajinasiri, Z. Hossaini and F. Rostami-Charati, Heteroat. Chem., 2011, 22, 625-629.

35 B. S. Babu and K. K. Balasubramanian, Tetrahedron Lett., 1998, 39, 9287-9288.

36 P. A. Grieco, J. J. Nunes and M. D. Gaul, J. Am. Chem. Soc., 1990, 112, 4595-4596.

37 A. Heydari, Tetrahedron, 2002, 58, 6777-6793.

38 Y. Pocker and G. T. Spyridis, J. Am. Chem. Soc., 2002, 124, 7390-7394.

39 S. Sankararaman and J. E. Nesakumar, Eur. J. Org. Chem., 2000, 2003-2011.

40 R. Sudha, K. Malola Narasimhan, V. Geetha Saraswathy and S. Sankararaman, J. Org. Chem., 1996, 61, 1877-1879.

41 D. J. Eyckens, M. E. Champion, B. L. Fox, P. Yoganantharajah, Y. Gibert, T. Welton and L. C. Henderson, Eur. J. Org. Chem., 2016, 913-917.

42 D. J. Eyckens, B. Demir, T. R. Walsh, T. Welton and L. C. Henderson, Phys. Chem. Chem. Phys., 2016, 18, 13153-13157.

43 P. Yoganantharajah, D. J. Eyckens, J. L. Pedrina, L. C. Henderson and Y. Gibert, New J. Chem., 2016, 40, 6599-6603. 